\title{
La salud de mujeres que viven cerca de pozos y estaciones de petróleo en la Amazonía ecuatoriana
}

\author{
Miguel San Sebastián, ${ }^{1}$ Ben Armstrong ${ }^{2}$ y Carolyn Stephens ${ }^{2}$
}

RESUMEN Objetivos. Investigar las condiciones ambientales y el estado de salud de las mujeres que viven en comunidades rurales rodeadas por pozos y estaciones de petróleo en la Amazonía del Ecuador.

Método. Se aplicó un diseño transversal comparativo, asignándose la exposición según la localización geográfica de las comunidades respecto a los pozos y estaciones de petróleo. Se analizaron muestras de agua de los ríos locales para determinar el contenido de hidrocarburos totales de petróleo y se proporcionó un cuestionario estructurado a cada cabeza de familia. El estudio se localizó en comunidades campesinas del nordeste del Ecuador y duró desde noviembre de 1998 hasta abril de 1999. Se incluyeron en el estudio nueve comunidades en el área expuesta (368 participantes) y 14 en el área no expuesta (291 participantes).

Resultados. Los ríos de las comunidades expuestas presentaron niveles de contaminación muy superiores al limite aceptado para el uso humano.

Se encontraron diferencias significativas en las razones de prevalencia de hongos en la piel (RP 1,37; IC95\% 1,01 a 1,86) en las dos semanas previas al estudio y de irritación de la nariz (RP 2,18; IC95\% 1,64 a 2,91) y garganta (RP 1,68; IC95\% 1,02 a 2,75) en los 12 meses anteriores. También se encontraron asociados con la exposición en las dos semanas previas el cansancio y "otros sintomas," y en los 12 meses anteriores, el dolor de cabeza, irritación ocular, dolor de oídos, diarrea y gastritis.

Conclusiones. Los síntomas observados en las participantes de las comunidades expuestas concuerdan con los síntomas de toxicidad causados por el petróleo. Hay una necesidad urgente de establecer un adecuado programa de control y remediación ambiental que evite este innecesario e inaceptable riesgo para la salud de estas poblaciones.

Palabras clave Salud de la mujer, salud ambiental, toxicidad por petróleo, hidrocarburos.

Desde 1972, compañías petroleras internacionales encabezadas por Texaco, en colaboración con la compañía nacio-

\footnotetext{
1 Instituto de Epidemiología y Salud Comunitaria "Manuel Amunárriz", Apdo. 17-10-7410 Nicolás López 349 y Avenida La Prensa, Quito, Ecuador. Tel.: (02) 257689; Fax: (02) 449763; Correo electrónico: sandyura@ecuanex.net.ec

2 Environmental Epidemiology Unit, London School of Hygiene and Tropical Medicine, Londres, Inglaterra, Reino Unido.
}

nal, Petroecuador, han extraído más de 2000 millones de barriles de petróleo del Ecuador, principalmente de la región amazónica. En este proceso, miles de millones de galones de petróleo y desechos tóxicos han sido eliminados directamente en el medio ambiente $(1,2)$.

Desde el inicio de la explotación petrolera, las poblaciones residentes en las áreas de producción de petróleo han expresado su preocupación ante la contaminación producida por esa práctica. Las comunidades indígenas y campesinas, así como grupos nacionales de ecologistas, se han organizado en clara oposición a la falta de regulación del desarrollo petrolero y han declarado que la contaminación causa un enorme daño tanto al medio ambiente como a la salud de la población $(2,3)$. Tanto campesinos como indígenas han informado que muchos esteros y ríos 
locales, antes ricos en peces, carecen hoy de vida acuática; también se registran habitualmente muertes de ganado por beber en ellos. Los residentes de las zonas de producción petrolera se quejan con frecuencia de los picores y sarpullidos que les produce bañarse en esas aguas $(1,3)$.

En 1993, una asociación local de promotores de salud hizo un estudio sobre los efectos del petróleo en la salud, en el que se incluyeron comunidades expuestas a la contaminación y comunidades donde no había explotación petrolera. Los habitantes de las comunidades expuestas presentaron más enfermedades (anemia, malnutrición, infecciones de la piel), abortos y una mayor tasa de mortalidad que los residentes de las comunidades no expuestas (4). En 1994, el Centro de Derechos Económicos y Sociales de Quito publicó un informe documentando los peligrosos niveles de contaminación por petróleo en los ríos del nordeste ecuatoriano. Las concentraciones de hidrocarburos aromáticos policíclicos en las aguas utilizadas para beber, bañarse o pescar fueron de 10 a 10000 veces superiores a los límites permitidos por la Agencia para la Protección Ambiental de los Estados Unidos de América. En ese mismo informe se destacaron las dermatosis que afectan a la población local y que están aparentemente relacionadas con la contaminación petrolera (5).

El desarrollo de la actividad petrolera en cada fase de su ciclo puede producir efectos adversos en el medio ambiente y en las poblaciones, desde los estudios sísmicos iniciales, la perforación, la producción del petróleo, el transporte y finalmente su procesamiento en las refinerías $(1,6)$. Los estudios sobre el impacto de esas actividades en la salud de las poblaciones locales se han concentrado en investigar los derrames de crudo en el mar (7-11) y en determinar el riesgo para la salud de las poblaciones radicadas en las cercanías de refinerías e industrias petroquímicas (12-14). Sin embargo, hay poca información sobre el impacto en la salud de las poblaciones que viven en las cercanías de los pozos y estaciones de petróleo.
Este estudio presenta las condiciones ambientales y de salud de varias comunidades de campesinos que viven en la proximidad de pozos y estaciones de petróleo en la Amazonía del Ecuador. La investigación es parte de un estudio más amplio que evalúa el impacto de la contaminación petrolera en la salud de las poblaciones rurales del país (15).

\section{MATERIALES Y MÉTODOS}

\section{Zona de estudio y población}

El estudio se concentró en varias comunidades campesinas situadas en los cantones de Orellana y Sachas en la provincia de Orellana y en el cantón Shushufindi en la provincia de Sucumbíos, ambas en el nordeste del Ecuador (figura 1). Las comunidades se eligieron por el largo período de actividad de las compañías petroleras en esa zona. En el nordeste ecuatoriano, las comunidades campesinas son grupos de familias con poblaciones que oscilan entre 100 y 400 personas. Cada campesino puede poseer hasta 50 hectáreas de terreno. En la zona estudiada viven aproximadamente 50000 campesinos, muchos de ellos a poca distancia de pozos y estaciones de petróleo. La mayor parte de las comunidades carecen de electricidad y agua entubada y tienen gran dificultad de acceso a los servicios de salud.

\section{Evaluación ambiental}

Para determinar si las comunidades cercanas a los pozos y estaciones de petróleo estaban expuestas a algún tipo de contaminación, durante los meses de febrero a abril de 1999 se recogieron muestras de las corrientes donde los residentes obtienen agua para beber, bañarse o lavar la ropa. Las muestras se tomaron en la temporada de invierno, cuando la presencia de petróleo no era visible en los ríos.

Se analizó el contenido de hidrocarburos totales de petróleo (HTP) en el agua en el laboratorio de aguas y suelos del colegio P. Miguel Gamboa, en
Coca. El método para medir los HTP consistió en la extracción con 1,1,2-tricloro-trifluor-etano y la determinación por espectrofotometría con rayos infrarrojos (límite de detección 0,001) (16). Los técnicos del laboratorio desconocían la procedencia de las muestras de agua en el momento del análisis. Por limitaciones técnicas y económicas, no fue posible medir también la contaminación de la tierra y el aire en las zonas de estudio.

\section{Selección de las comunidades}

Se seleccionaron dos grupos de comunidades para el estudio: comunidades donde la población vivía expuesta a la contaminación proveniente de los pozos y estaciones de petróleo, y comunidades no expuestas seleccionadas como grupo testigo. Las comunidades del grupo testigo comprendieron una población con características geográficas y sociodemográficas similares a las del área expuesta (17). Se definieron como comunidades expuestas aquellas comunidades localizadas dentro de un área de $5 \mathrm{~km}$ alrededor de un pozo o estación de petróleo, siguiendo el río a favor de la corriente. Las comunidades no expuestas se definieron como aquellas comunidades que se encontraban a una distancia mínima de $30 \mathrm{~km}$ en contra de la corriente del río. Todas las comunidades estudiadas se encontraban lejos de cualquier otra industria química.

\section{Selección y cálculo de la muestra}

El procedimiento de muestreo se llevó a cabo en dos fases. Primero, se elaboró una lista de todas las comunidades de la zona, cada una de las cuales se clasificó como expuesta o no expuesta. En segundo lugar, se seleccionó al azar un grupo de comunidades y, en ellas, a todas las mujeres de edades comprendidas entre los $17 \mathrm{y}$ los 45 años que hubieran residido al menos tres años en la comunidad. Estos criterios de inclusión se basaron en considerar a esta población vulnerable a los contaminantes del petróleo 
FIGURA 1. Mapa de la zona de estudio, nordeste del Ecuador

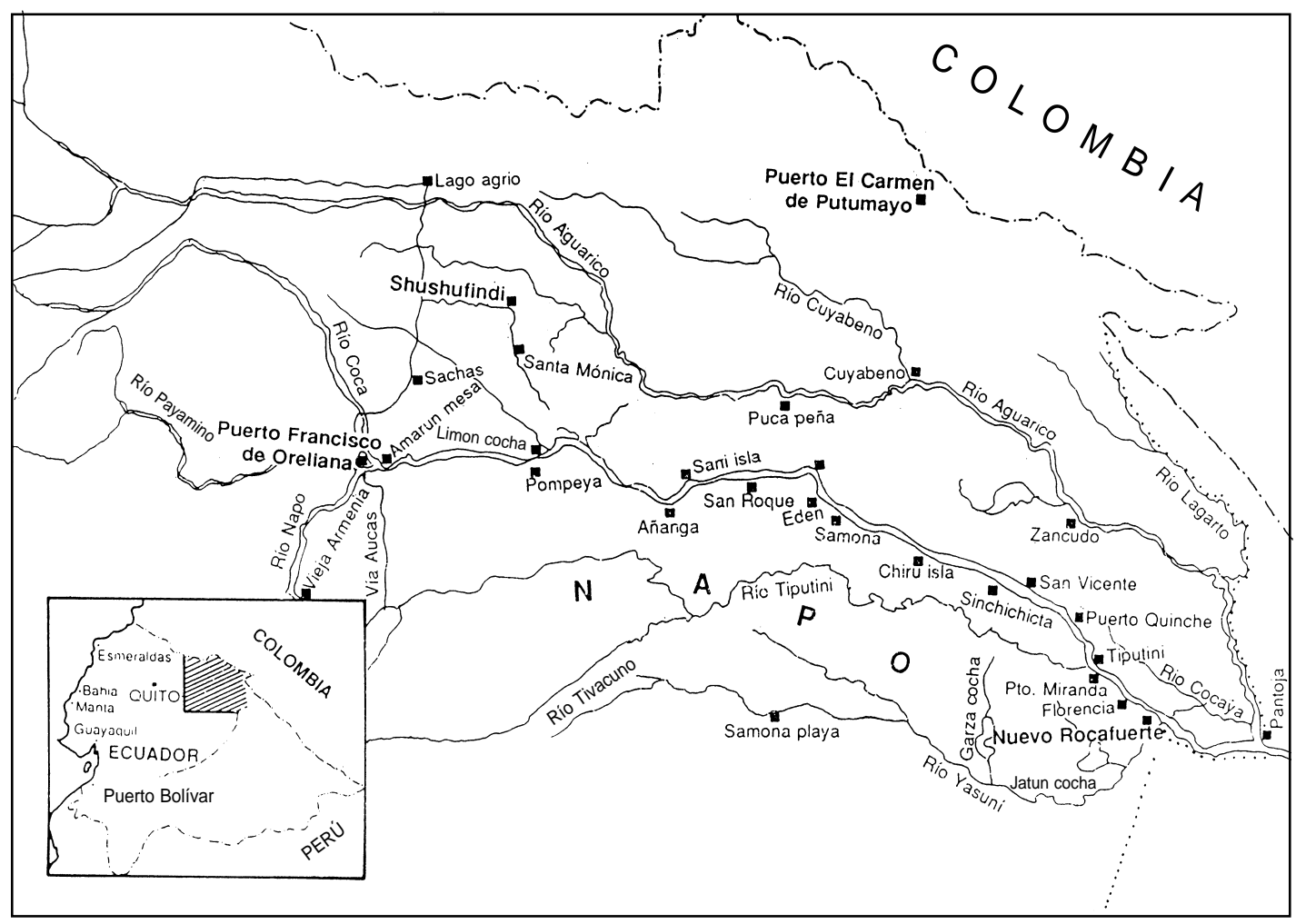

debido a sus actividades diarias. Además, era fácil de contactar por trabajar principalmente en la casa. El criterio de haber vivido al menos tres años en la misma comunidad se escogió como una medida aproximada que indicara exposición crónica.

Los líderes de las comunidades colaboraron identificando a las mujeres que cumplían los criterios de selección y solicitando su participación en el estudio. El tamaño de la muestra se calculó de acuerdo con el número de mujeres necesario para detectar una diferencia en la prevalencia del doble entre los grupos expuesto y no expuesto a un nivel de significación de $5 \%$ y con un poder de $80 \%$, dando por sentada una prevalencia de síntomas de $20 \%$ en el grupo testigo. El tamaño de la muestra se duplicó como ajuste por la naturaleza del agrupamiento del muestreo. El cálculo se realizó con el programa Epi Info 6, que indicó un requisito de 182 mujeres por grupo. Dando por sentada una participación de $70 \%$, el tamaño de la muestra se incrementó a 260 mujeres en cada grupo para obtener así una estimación más real. En total, se incluyeron en el estudio nueve comunidades expuestas y 14 no expuestas.

\section{Recolección de datos}

El trabajo de campo se realizó entre los meses de noviembre de 1998 y abril de 1999. Se preparó un cuestionario estructurado para administrar a la cabeza de familia de cada domicilio. El cuestionario constó de dos partes. En la primera parte se registraron las características demográficas (edad, grupo étnico, tiempo de residencia, estado civil) y socioeconómicas (grado de escolaridad, ocupación de la mujer, ocupación de su pareja y condiciones de la vivienda) de la población. En la segunda parte se recogió información sobre la historia médica de las mujeres que participaron en el estudio y su uso de los servicios de salud durante las 2 semanas y los 12 meses anteriores al estudio. En el caso de la historia médica, las participantes debían revelar si habían tenido alguno de 23 síntomas enumerados respondiendo a las siguientes preguntas: “ ¿Ha tenido usted alguno de estos síntomas en las últimas 2 semanas?" “¿Ha tenido usted alguno de estos síntomas en los últimos 12 meses?". Durante la entrevista, no se dieron definiciones de síntomas ni tampoco se realizaron exámenes médicos. Esta parte del cuestionario se adaptó de otros estudios comunitarios $(4,8)$.

Un asistente que residía fuera de la zona de estudio colaboró en la recogida de datos y asistió a una semana de entrenamiento, en la que se realizaron prácticas de entrevistas. Tanto el investigador como el asistente fueron conscientes del estado de exposición de las comunidades, ya que era imposible evitarlo. El cuestionario se probó en una de las comunidades con objeto de descubrir posibles problemas de 
comprensión y administración. Los resultados de esta experiencia se discutieron y se realizaron los cambios necesarios.

A las mujeres seleccionadas para el estudio se les pidió que colaboraran participando en una entrevista personal. La investigación se describió como un estudio para evaluar su estado general de salud. Se acordó una fecha de reunión con las participantes en el centro de cada comunidad, donde fueron entrevistadas en una habitación privada de la escuela o de la casa comunitaria. La primera parte del cuestionario la realizó el investigador y la segunda, el asistente. La entrevista duró aproximadamente 30 minutos.

Antes de realizar el estudio, se obtuvo el consentimiento informado de todas las participantes. Toda la información recogida se consideró confidencial y las personas enfermas recibieron atención médica y tratamiento de forma gratuita. Al término de la investigación, se informó a las comunidades de los resultados.

\section{Análisis de los datos}

El primer análisis de los datos se realizó con el programa Epi Info, versión 6, y posteriormente se utilizó el programa Stata, versión 5, para análisis más complejos. Se calcularon las razones de prevalencia (RP) de los distintos síntomas con un intervalo de confianza de 95\% (IC95\%). ${ }^{3}$ Posteriormente, por medio de análisis de regresión logística, se calcularon las razones de prevalencia de dichos trastornos, ajustados según los posibles factores de confusión. La edad y el nivel socioeconómico (educación, ocupación de la mujer y su pareja y condiciones de la vivienda) se examinaron como factores

\footnotetext{
Cox y Snell (18) recomiendan una $P>0,1$ para indicar una diferencia estadísticamente significativa debido a que la mayor parte de los estudios epidemiológicos sobre asuntos ambientales solo son capaces de encontrar riesgos moderados, que no dejan de ser importantes desde el punto de vista de la salud pública si un gran número de personas está expuesto, como en el caso del presente estudio $(19,20)$.
}

de confusión potenciales por ser determinantes importantes del estado de salud (21). Durante el proceso de análisis, los errores estándar se ajustaron también por la naturaleza del agrupamiento del muestreo usando el método de Huber-White (22).

\section{RESULTADOS}

\section{Evaluación del medio ambiente}

Los resultados de los análisis se presentan en el cuadro 1. Se investigaron 20 ríos pertenecientes a las nueve comunidades expuestas. De ellos, 18 ríos en ocho comunidades estaban contaminados con HTP, en concentraciones que variaron de 0,02 partes por millón (ppm) en el río Manduro 1 hasta 2,883 ppm en el río Basura. En algunos ríos, la concentración de hidrocarburos superó más de 100 veces el límite permitido para consumo humano según las

CUADRO 1. Concentración de hidrocarburos totales de petróleo (HTP) a en los ríos de las comunidades cercanas a los pozos petroleros, Ecuador, 1998-1999

\begin{tabular}{|c|c|c|}
\hline \multicolumn{2}{|c|}{ Identificación (ríos) } & $\begin{array}{l}\text { Hidrocarburos } \\
\text { totales de } \\
\text { petróleo (ppm) }\end{array}$ \\
\hline \multirow[t]{2}{*}{ Comunidad 1} & Toachi & 0 \\
\hline & Escuela 28-M & 0 \\
\hline \multirow[t]{2}{*}{ Comunidad 2} & Pozo 66 & 0,04 \\
\hline & Río Negro & 1,438 \\
\hline \multirow[t]{2}{*}{ Comunidad 3} & Victoria 1 & 0,051 \\
\hline & Victoria 2 & 1,426 \\
\hline \multirow[t]{2}{*}{ Comunidad 4} & Itaya 1 & 0,043 \\
\hline & Itaya 2 & 0,028 \\
\hline \multirow[t]{2}{*}{ Comunidad 5} & Escuela 18-N & 0,036 \\
\hline & Jiménez & 0,028 \\
\hline \multirow[t]{4}{*}{ Comunidad 6} & Huamayacu & 1,444 \\
\hline & Basura & 2,883 \\
\hline & Iniap & 0,097 \\
\hline & Huamayacu verde & 0,529 \\
\hline \multirow[t]{2}{*}{ Comunidad 7} & Lumu pueblo & 0,066 \\
\hline & Lumu 3 & 0,055 \\
\hline Comunidad 8 & Dayuma & 0,145 \\
\hline \multirow[t]{3}{*}{ Comunidad 9} & Manduro 1 & 0,02 \\
\hline & Piscina Manduro & 0,434 \\
\hline & Manduro 2 & 0,108 \\
\hline
\end{tabular}

regulaciones de la Comunidad Europea (16).

\section{Características de las participantes}

Las tasas de participación fueron de $70,2 \%$ en la zona expuesta (428 mujeres) y $79,0 \%$ en la no expuesta $(347 \mathrm{mu}-$ jeres). Sesenta mujeres $(14,0 \%)$ en el área expuesta y $56(16,1 \%)$ en la no expuesta fueron excluidas del estudio por tener menos de 3 años de residencia en las comunidades. En total, se dispuso de información sobre 368 y 291 mujeres, respectivamente.

Entre las participantes de las comunidades expuestas había pocas diferencias con respecto al tiempo de residencia en la comunidad, la edad, el grupo étnico, el estado civil y el grado de escolaridad en comparación con las mujeres del área no expuesta (cuadro 2). Sin embargo, las mujeres de las áreas expuestas trabajaban menos en la agricultura $(72,6 \%)$ que las del área no expuesta $(86,3 \%)$. En las comunidades expuestas, los maridos tendieron a trabajar más en las compañías petroleras $(7,8 \%)$ que en el grupo testigo $(1,3 \%)$. Las casas de cemento y las letrinas, así como la presencia de una refrigeradora en el hogar, fueron más frecuentes en las comunidades expuestas, lo que indica un nivel económico más alto.

\section{Condiciones de salud}

En las dos semanas antes del estudio, se encontró una diferencia significativa estadísticamente en la prevalencia de hongos en la piel después de ajustar por los factores de confusión (RP 1,37; IC95\% 1,01 a 1,86). La presencia de otros síntomas (RP 1,33; IC $95 \%$ 0,97 a 1,83) y de cansancio (RP 1,48; IC95\% 0,94 a 2,30) se encontró también asociada con la exposición (cuadro 3).

En los 12 meses que precedieron al estudio, la prevalencia de irritación nasal (RP 2,18; IC95\% 1,64 a 2,91) y de la garganta (RP 1,68; IC95\% 1,02 a 2,75) en ambos grupos mostró diferencias 
estadísticamente significativas después de ajustar por los factores de confusión. El dolor de cabeza (RP 1,56; IC95\% 0,90 a 2,71), la irritación de los ojos (RP 1,28; IC95\% 0,97 a 1,69), el dolor de oídos (RP 1,32; IC95\% 0,95 a 1,84), la diarrea (RP 1,54; IC95\% 0,94 a 2,51 ) y la gastritis (RP 1,43; IC95\% 0,92 a 2,22 ) se encontraron también asociados con la cercanía a pozos y estaciones de petróleo (cuadro 4).

El cuadro 5 presenta la prevalencia de los trastornos físicos y el uso de los servicios de salud notificados por las participantes. Después del ajuste por los factores de confusión, no se encontraron diferencias significativas entre los dos grupos en los indicadores utilizados.

\section{DISCUSIÓN}

Este estudio presenta un análisis de la contaminación ambiental y del estado de salud de las mujeres que vivían durante el período de estudio en comunidades próximas a pozos y estaciones de petróleo en la Amazonía ecuatoriana. El análisis del agua mostró intensa exposición a los compuestos químicos del petróleo entre los residentes de las comunidades expuestas. Los datos obtenidos confirman que los habitantes de esas comunidades estaban sometidos a contaminantes provenientes de la actividad petrolera que exceden con mucho los límites de seguridad reconocidos internacionalmente. Aunque no se conoce desde cuándo la población ha estado expuesta a esos riesgos, numerosos informes señalan que la contaminación se inició con la explotación petrolera en los años setenta $(1,5,23,24)$.

En 1994, un estudio realizado por el Centro de Derechos Económicos y Sociales del Ecuador mostró concentraciones elevadas de contaminantes derivados del petróleo en los ríos de la zona de estudio, lo que evidencia la larga exposición de los residentes a esos productos tóxicos (5). Recientemente, el Ministerio de Medio Ambiente apoyó los resultados de ese estudio informando que en los ríos de

CUADRO 2. Características sociodemográficas de las participantes en el estudio ( $\bar{x}$ = media; de = desviación estándar), Ecuador, 1998-1999

\begin{tabular}{|c|c|c|c|c|}
\hline Característica & \multicolumn{2}{|c|}{$\begin{array}{c}\text { Grupo expuesto (\%) } \\
n=368\end{array}$} & \multicolumn{2}{|c|}{$\begin{array}{c}\text { Grupo no expuesto (\%) } \\
n=291\end{array}$} \\
\hline $\begin{array}{l}17-20 \\
21-25 \\
26-30 \\
31-35 \\
36-40 \\
41-45\end{array}$ & $\begin{array}{c}45 \\
72 \\
75 \\
65 \\
56 \\
55 \\
\bar{x}=30,5\end{array}$ & $\begin{array}{c}(12,2) \\
(19,6) \\
(20,4) \\
(17,7) \\
(15,2) \\
(14,9) \\
\text { de }=7,8\end{array}$ & $\begin{array}{c}43 \\
45 \\
63 \\
43 \\
49 \\
48 \\
\bar{x}=30,8\end{array}$ & $\begin{array}{c}(14,8) \\
(15,5) \\
(21,6) \\
(14,8) \\
(16,8) \\
(16,5) \\
\mathrm{de}=8,3\end{array}$ \\
\hline $\begin{array}{l}\text { Tiempo de residencia (años) } \\
4-10 \\
11-20 \\
>21\end{array}$ & $\begin{array}{c}116 \\
191 \\
61 \\
\bar{x}=14,4\end{array}$ & $\begin{array}{c}(31,5) \\
(51,9) \\
(16,6) \\
\text { de }=6,1\end{array}$ & $\begin{array}{c}112 \\
147 \\
32 \\
\bar{x}=13,5\end{array}$ & $\begin{array}{c}(38,5) \\
(50,5) \\
(11,0) \\
\text { de }=6,1\end{array}$ \\
\hline $\begin{array}{l}\text { Grupo étnico: } \\
\text { Mestizo } \\
\text { Negro } \\
\text { Indígena }\end{array}$ & $\begin{array}{r}360 \\
6 \\
2\end{array}$ & $\begin{array}{r}(97,8) \\
(1,6) \\
(0,5)\end{array}$ & $\begin{array}{r}283 \\
2 \\
6\end{array}$ & $\begin{array}{r}(97,3) \\
(0,7) \\
(2,1)\end{array}$ \\
\hline $\begin{array}{l}\text { Estado civil: } \\
\text { Soltera } \\
\text { Casada } \\
\text { Viuda }\end{array}$ & $\begin{array}{r}38 \\
308 \\
22\end{array}$ & $\begin{array}{r}(10,3) \\
(83,7) \\
(6,0)\end{array}$ & $\begin{array}{r}30 \\
245 \\
16\end{array}$ & $\begin{array}{r}(10,3) \\
(84,2) \\
(5,5)\end{array}$ \\
\hline $\begin{array}{l}\text { Educación: } \\
\text { Ninguna } \\
\text { Primaria no terminada } \\
\text { Primaria } \\
\text { Secundaria no terminada } \\
\text { Secundaria }\end{array}$ & $\begin{array}{r}17 \\
74 \\
216 \\
41 \\
20\end{array}$ & $\begin{array}{r}(4,6) \\
(20,1) \\
(58,7) \\
(11,1) \\
(5,4)\end{array}$ & $\begin{array}{r}8 \\
69 \\
178 \\
28 \\
8\end{array}$ & $\begin{array}{r}(2,7) \\
(23,7) \\
(61,2) \\
(9,6) \\
(2,7)\end{array}$ \\
\hline $\begin{array}{l}\text { Total de personas en casa: } \\
\text { Principal ocupación: } \\
\text { Agricultura } \\
\text { Otra }\end{array}$ & $\begin{array}{l}\bar{x}=6,3 \\
267 \\
101\end{array}$ & $\begin{array}{c}\text { de }=4,5 \\
(72,6) \\
(27,4)\end{array}$ & $\begin{array}{c}\bar{x}=6,1 \\
251 \\
40\end{array}$ & $\begin{array}{c}\text { de }=2,4 \\
(86,3) \\
(13,8)\end{array}$ \\
\hline $\begin{array}{l}\text { Ocupación del marido: } \\
\text { Agricultura } \\
\text { Compañía petrolera } \\
\text { Palma africana } \\
\text { Otra }\end{array}$ & $\begin{array}{r}243 \\
29 \\
9 \\
27\end{array}$ & $\begin{array}{r}(66,0) \\
(7,8) \\
(2,4) \\
(7,3)\end{array}$ & $\begin{array}{r}223 \\
4 \\
3 \\
16\end{array}$ & $\begin{array}{r}(76,6) \\
(1,4) \\
(1,0) \\
(5,5)\end{array}$ \\
\hline $\begin{array}{l}\text { Condiciones de la vivienda: } \\
\text { Casa de cemento } \\
\text { Refrigerador } \\
\text { Letrina }\end{array}$ & $\begin{array}{r}49 \\
127 \\
186\end{array}$ & $\begin{array}{l}(13,3) \\
(34,5) \\
(50,5)\end{array}$ & $\begin{array}{r}20 \\
39 \\
111\end{array}$ & $\begin{array}{r}(6,9) \\
(13,4) \\
(38,1)\end{array}$ \\
\hline
\end{tabular}

una de las comunidades de nuestro estudio se habían detectado concentraciones de HTP 500 veces superiores al límite considerado inocuo para el ser humano (25).

Los principales problemas de salud de las participantes de las comunidades expuestas, después de ajustar por los factores de confusión, fueron los hongos en la piel, otros síntomas y cansancio en las dos semanas anterio- res al estudio. También habían sufrido irritación nasal, dolor de garganta y de la cabeza, irritación de los ojos, dolor de oídos, diarrea y gastritis en los 12 meses previos a la encuesta.

Los principales efectos hallados en otros estudios de exposición aguda al petróleo tras derrames de petróleo en el mar han sido dolor de cabeza, irritación de la garganta, irritación de los ojos y cansancio $(8,10)$. En una de las 
CUADRO 3. Prevalencia de síntomas referidos por las mujeres de las comunidades expuestas y no expuestas en las dos semanas anteriores a la encuesta con sus correspondientes razones de prevalencia (RP) no ajustadas, intervalos de confianza de $95 \%$ (IC95\%) y razones de prevalencia ajustadas, intervalos de confianza de $95 \%$ y valores $P,{ }^{a}$ Ecuador, $1998-1999$

\begin{tabular}{|c|c|c|c|c|c|c|c|c|c|}
\hline \multirow[b]{2}{*}{ Síntomas } & \multicolumn{2}{|c|}{$\begin{array}{c}\text { Comunidades } \\
\text { expuestas }\end{array}$} & \multicolumn{2}{|c|}{$\begin{array}{l}\text { Comunidades } \\
\text { no expuestas }\end{array}$} & \multirow[b]{2}{*}{$\mathrm{RP}$} & \multirow[b]{2}{*}{ IC95\% } & \multirow{2}{*}{$\begin{array}{c}\mathrm{RP}^{\mathrm{b}} \\
\text { ajustada }\end{array}$} & \multirow[b]{2}{*}{ IC95\% } & \multirow{2}{*}{$\begin{array}{l}\text { Valor } \\
P\end{array}$} \\
\hline & No & $\%$ & No & $\%$ & & & & & \\
\hline \multicolumn{10}{|l|}{ Generales: } \\
\hline Fiebre & 94 & $(25,5)$ & 58 & $(19,9)$ & 1,37 & $0,87-2,16$ & 1,32 & $0,82-2,13$ & $>0,20$ \\
\hline Dolor de cabeza & 279 & $(75,8)$ & 226 & $(77,7)$ & 0,90 & $0,64-1,26$ & 1,01 & $0,70-1,45$ & $>0,20$ \\
\hline Ojos irritados & 179 & $(48,6)$ & 131 & $(45,0)$ & 1,15 & $0,90-1,47$ & 1,12 & $0,80-1,55$ & $>0,20$ \\
\hline Mareo & 229 & $(62,2)$ & 168 & $(57,7)$ & 1,20 & $0,76-1,90$ & 1,41 & $0,89-2,23$ & 0,14 \\
\hline \multicolumn{10}{|l|}{ Respiratorios: } \\
\hline Irritación nasal & 141 & $(38,3)$ & 105 & $(36,1)$ & 1,10 & $0,81-1,49$ & 1,14 & $0,84-1,55$ & $>0,20$ \\
\hline Tos & 214 & $(58,2)$ & 157 & $(54,0)$ & 1,18 & $0,89-1,57$ & 1,15 & $0,80-1,65$ & $>0,20$ \\
\hline Bronquitis & 11 & $(3,0)$ & 11 & $(3,8)$ & 0,78 & $0,36-1,69$ & 0,73 & $0,27-1,93$ & $>0,20$ \\
\hline \multicolumn{10}{|l|}{ Digestivos: } \\
\hline Gastritis & 266 & $(72,3)$ & 192 & $(66,0)$ & 1,34 & $0,89-2,02$ & 1,27 & $0,79-2,04$ & $>0,20$ \\
\hline Dolor en la piel & 235 & $(63,9)$ & 174 & $(59,8)$ & 1,18 & $0,82-1,72$ & 1,21 & $0,80-1,83$ & $>0,20$ \\
\hline \multicolumn{10}{|l|}{ De la piel: } \\
\hline Piel roja & 72 & $(19,6)$ & 63 & $(21,6)$ & 0,88 & $0,52-1,47$ & 1,02 & $0,57-1,81$ & $>0,20$ \\
\hline Piodermitis & 81 & $(22,0)$ & 63 & $(21,6)$ & 1,02 & $0,61-1,69$ & 1,07 & $0,61-1,86$ & $>0,20$ \\
\hline Hongos & 143 & $(38,9)$ & 88 & $(30,2)$ & 1,46 & $1,08-1,97$ & 1,37 & $1,01-1,86$ & 0,03 \\
\hline \multicolumn{10}{|l|}{ Musc.-esquel: } \\
\hline Dolor cuerpo & 239 & $(64,9)$ & 190 & $(65,3)$ & 0,98 & $0,66-1,45$ & 1,13 & $0,73-1,74$ & $>0,20$ \\
\hline Dolor articulac. & 204 & $(55,4)$ & 178 & $(61,2)$ & 0,79 & $0,50-1,22$ & 0,82 & $0,49-1,39$ & $>0,20$ \\
\hline Calambres & 94 & $(25,5)$ & 90 & $(30,9)$ & 0,76 & $0,49-1,18$ & 0,87 & $0,54-1,39$ & $>0,20$ \\
\hline \multicolumn{10}{|l|}{ Nerviosos: } \\
\hline Problemas para dormir & 138 & $(37,5)$ & 98 & $(33,7)$ & 1,18 & $0,80-1,74$ & 1,32 & $0,83-2,13$ & $>0,20$ \\
\hline
\end{tabular}

a Errores estándar ajustados para el agrupamiento del muestreo.

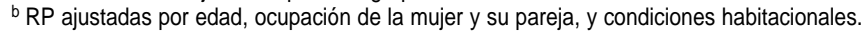

poblaciones que tuvo esa experiencia, el grupo expuesto mostró también más ansiedad y depresión que el no expuesto (10). Otra población que participó recientemente en las tareas de limpieza de un derrame de crudo en las costas de Japón registró un aumento en la prevalencia de síntomas como el dolor de espalda, de piernas y de cabeza, así como irritación de los ojos y de la garganta, con respecto a los días previos al derrame (11).

En nuestro estudio, la mayor prevalencia de los síntomas hallados en la población expuesta podría explicarse por la exposición ambiental al petróleo mediante su ingestión, inhalación o contacto con la piel. El contacto acci- dental con el petróleo o la exposición a sus vapores puede causar irritación de la piel o de los ojos. Una exposición prolongada o repetida a bajas concentraciones de los componentes volátiles del petróleo puede producir náusea, mareos y dolor de cabeza $(26,27)$.

En nuestro estudio, las participantes de las comunidades expuestas revelaron síntomas similares a los de grupos que han sido expuestos a derrames accidentales de petróleo (8-11) y que han sufrido los efectos tóxicos atribuidos al petróleo (10). Por las razones anteriores, cabría esperar una diferencia mayor entre las prevalencias calculadas para la población expuesta y la no expuesta. Sin embargo, las pequeñas diferencias en la prevalencia de los síntomas de ambas poblaciones podrían explicarse por lo siguiente. En primer lugar, la prevalencia de los síntomas experimentados por ambas poblaciones es muy alta (28), lo que podría reflejar el limitado acceso a los servicios de salud y las difíciles condiciones de vida en la selva. Segundo, diferentes estudios han mostrado la relación que existe entre un nivel socioeconómico bajo y un mal estado de salud (29-31). En nuestro estudio, la población expuesta podría verse beneficiada económicamente por la existencia de la industria petrolera (como sugieren los indicadores socioeconómicos utilizados), lo cual quizá ayudó a disminuir 
CUADRO 4. Prevalencia de síntomas referidos por las mujeres de las comunidades expuestas y no expuestas en los 12 meses anteriores a la encuesta con sus correspondientes razones de prevalencia (RP) no ajustadas, intervalos de confianza de $95 \%$ (IC95\%) y razones de prevalencia ajustadas, intervalos de confianza de $95 \%$ y valores $P$, a Ecuador, 1998-1999

\begin{tabular}{|c|c|c|c|c|c|c|c|c|c|}
\hline \multirow[b]{2}{*}{ Síntomas } & \multicolumn{2}{|c|}{$\begin{array}{l}\text { Comunidades } \\
\text { expuestas }\end{array}$} & \multicolumn{2}{|c|}{$\begin{array}{l}\text { Comunidades } \\
\text { no expuestas }\end{array}$} & \multirow[b]{2}{*}{$\mathrm{RP}$} & \multirow[b]{2}{*}{ IC95\% } & \multirow{2}{*}{$\begin{array}{c}\mathrm{RPb}^{\mathrm{b}} \\
\text { ajustada }\end{array}$} & \multirow[b]{2}{*}{ IC95\% } & \multirow{2}{*}{$\begin{array}{l}\text { Valor } \\
P\end{array}$} \\
\hline & No & $\%$ & No & $\%$ & & & & & \\
\hline \multicolumn{10}{|l|}{ Generales: } \\
\hline Fiebre & 226 & $(61,4)$ & 172 & $(59,1)$ & 1,10 & $0,75-1,61$ & 1,16 & $0,76-1,75$ & $>0,20$ \\
\hline Dolor cabeza & 319 & $(86,7)$ & 242 & $(83,2)$ & 1,31 & $0,83-2,08$ & 1,56 & $0,90-2,71$ & 0,10 \\
\hline Ojos irritados & 181 & $(49,2)$ & 128 & $(44,0)$ & 1,23 & $0,97-1,56$ & 1,28 & $0,97-1,69$ & 0,08 \\
\hline Mareo & 235 & $(63,9)$ & 176 & $(60,5)$ & 1,15 & $0,81-1,63$ & 1,35 & $0,86-2,12$ & 0,18 \\
\hline \multicolumn{10}{|l|}{ Respiratorios: } \\
\hline Irritación nasal & 170 & $(46,2)$ & 89 & $(30,6)$ & 1,94 & $1,47-2,57$ & 2,18 & $1,64-2,91$ & $<0,01$ \\
\hline Tos & 276 & $(75,0)$ & 196 & $(67,4)$ & 1,45 & $1,01-2,07$ & 1,35 & $0,86-2,12$ & 0,18 \\
\hline Bronquitis & 20 & $(5,4)$ & 13 & $(4,5)$ & 1,22 & $0,55-2,72$ & 1,40 & $0,56-3,46$ & $>0,20$ \\
\hline Gastritis & 263 & $(71,5)$ & 181 & $(62,2)$ & 1,52 & $1,04-2,21$ & 1,43 & $0,92-2,22$ & 0,10 \\
\hline Dolor en la piel & 252 & $(68,5)$ & 181 & $(62,2)$ & 1,32 & $0,87-1,99$ & 1,21 & $0,72-2,04$ & $>0,20$ \\
\hline \multicolumn{10}{|l|}{ Piel: } \\
\hline Piel roja & 95 & $(25,8)$ & 65 & $(22,3)$ & 1,21 & $0,73-1,99$ & 1,31 & $0,76-2,25$ & $>0,20$ \\
\hline Piodermitis & 100 & $(27,2)$ & 79 & $(27,1)$ & 1,0 & $0,60-1,65$ & 0,94 & $0,53-1,68$ & $>0,20$ \\
\hline Hongos & 147 & $(39,9)$ & 97 & $(33,3)$ & 1,33 & $0,99-1,78$ & 1,15 & $0,83-1,59$ & $>0,20$ \\
\hline \multicolumn{10}{|l|}{ Musc.-esquel: } \\
\hline Dolor cuerpo & 244 & $(66,3)$ & 179 & $(61,5)$ & 1,23 & $0,83-1,81$ & 1,26 & $0,77-2,06$ & $>0,20$ \\
\hline Dolor articulac. & 170 & $(46,2)$ & 128 & $(44,0)$ & 1,09 & $0,78-1,52$ & 1,09 & $0,74-1,61$ & $>0,20$ \\
\hline Calambres & 127 & $(34,5)$ & 101 & $(34,7)$ & 0,99 & $0,69-1,40$ & 1,10 & $0,76-1,59$ & $>0,20$ \\
\hline \multicolumn{10}{|l|}{ Nerviosos: } \\
\hline Problemas para dormir & 146 & $(39,7)$ & 100 & $(34,4)$ & 1,25 & $0,84-1,87$ & 1,39 & $0,88-2,20$ & 0,15 \\
\hline
\end{tabular}

a Errores estándar ajustados para el agrupamiento del muestreo.

${ }^{\mathrm{b}} \mathrm{RP}$ ajustadas por edad, ocupación de la mujer y su pareja, y condiciones de la habitacionales.

la prevalencia de síntomas en el grupo expuesto.

Este estudio refleja también las condiciones adversas de salud que prevalecen en las zonas rurales del Ecuador, donde la alta prevalencia de enfermedades se aúna con una escasa utilización de los servicios de salud. Con respecto a la morbilidad, la alta prevalencia de síntomas es similar a la que indican otros estudios comunitarios de salud en áreas rurales. Estudios realizados en Bolivia y en poblaciones indígenas de la Amazonía del Ecuador informan que $42 \%$ de los participantes habían experimentado algún problema de salud en las dos semanas previas al estudio $(28,32)$. En nuestro estudio, el uso de los servicios de salud fue similar en ambos grupos, lo cual indica que las diferencias de salud entre ellos no se deben a una diferente utilización de los servicios de salud.

A la hora de interpretar los resultados, varias consideraciones metodológicas deben tenerse en cuenta. La similitud de las variables sociodemográficas entre los grupos estudiados indica que las comunidades no expuestas fueron una población de referencia adecuada. El sesgo por falta de respuesta es siempre una posible fuente de error en todo estudio epidemiológico. Sin embargo, las tasas de participación fueron altas y similares en las áreas expuestas y no expuestas, lo cual $(70,2 \%$ y $79,0 \%)$ de alguna manera elimina ese tipo de sesgo. Esta alta participación se puede atribuir a la colaboración de los líderes de las comunidades. Las razones que tuvieron otras habitantes locales para no participar nos son desconocidas.

Un posible origen de sesgo en la selección de la muestra proviene de que solo las residentes actuales de las comunidades fueron elegidas para el estudio. En esta población, la emigración pudo haber sido un factor importante. Sin embargo, no pudo determinarse el impacto de ese sesgo.

También puede haber un sesgo en la recordación de los síntomas por parte de las participantes, especialmente al 
CUADRO 5. Prevalencia de trastornos físicos y uso de los servicios de salud referidos por las mujeres de las comunidades expuestas y no expuestas con sus correspondientes razones de prevalencia (RP) no ajustadas, intervalos de confianza de $95 \%$ (IC95\%) y razones de prevalencia ajustadas, intervalos de confianza de $95 \%$ y valores $P,{ }^{\text {a }}$ Ecuador, $1998-1999$

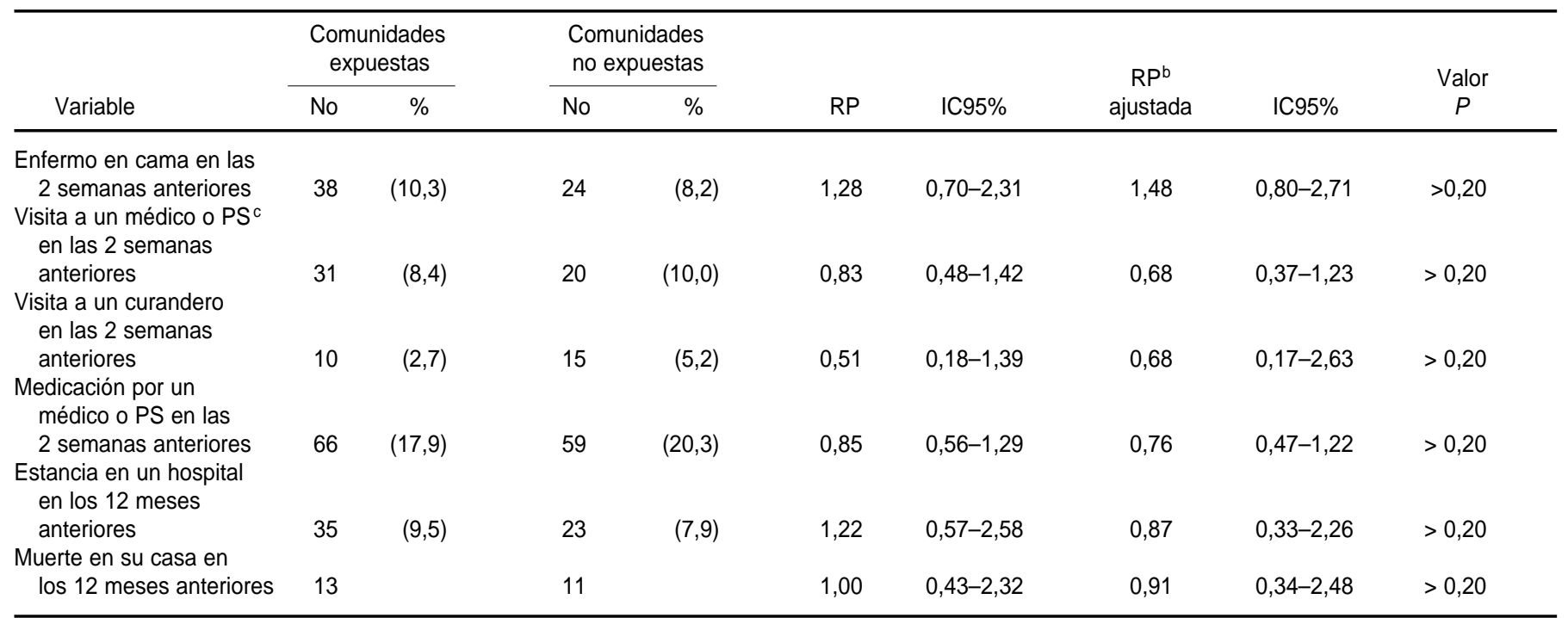

a Errores estándar ajustados para el agrupamiento del muestreo.

${ }^{b}$ RP ajustadas por edad, ocupación de la mujer y su pareja, y condiciones de la vivienda.

c Promotor de salud.

abarcar los 12 meses anteriores. Para la mayoría de las variables de estudios sobre salud comunitaria, se recomienda como razonable un período de recordación de dos o, a lo máximo, cuatro semanas (33). En nuestro estudio, a pesar del riesgo de que las mujeres no recordaran bien los síntomas, elegimos un período de recordación de 12 meses para poder evaluar la prevalencia de los síntomas en el contexto de una exposición crónica a los contaminantes. Por otro lado, la falta de registros médicos y la dificultad de verificar ciertas quejas, como el dolor de cabeza o de garganta, el cansancio, etc., podrían afectar a la validez de los resultados. También debe tenerse en cuenta el sesgo del entrevistador, ya que los investigadores eran conscientes de la exposición a que habían sido sujetas las comunidades.

Otra consideración es el sesgo de información, por el cual las personas que creen estar expuestas pueden exagerar la intensidad de los síntomas. Para disminuir este sesgo, en otros estudios se hacen ajustes por el aumento de ansiedad o la creencia de que la exposición podría afectar a su salud $(10,34)$. En nuestro estudio, no fue posible seguir esa práctica debido al largo período de exposición al petróleo de las comunidades participantes y la creencia entre los habitantes de esas comunidades de que el petróleo estaba desmejorando su salud (comunicación personal). Se intentó resolver esos problemas presentando la investigación a las comunidades como un estudio de su estado general de salud. Además, la especificidad de los síntomas más fuertemente asociados con la exposición sugerían un mecanismo causal entre la exposición y los síntomas, lo cual, en oposición a un "efecto pansintomático" (34), hace que este tipo de sesgo no sea una explicación adecuada de las diferencias observadas.

Uno de los factores que limitan los estudios sobre los efectos de las condiciones ambientales en la salud es la precisión con que se clasifican las comunidades expuestas y no expuestas. Algunas de esas consideraciones se tuvieron en cuenta al diseñar el estudio. Las comunidades a una distancia menor de $5 \mathrm{~km}$ de un pozo o estación de petróleo se consideraron expuestas. Las comunidades que consideramos no expuestas estaban situadas a un mínimo de $30 \mathrm{~km}$ en dirección río arriba de un pozo o estación.

En nuestro estudio, no existían datos de cómo la población había sido expuesta a los contaminantes derivados del petróleo en el pasado. Por otro lado, es escasa o nula la información sobre la toxicidad crónica de las diferentes sustancias químicas provenientes de los pozos y estaciones de petróleo. Se desconoce incluso el inventario de las sustancias químicas que derraman las compañías petroleras (1).

En el diseño del muestreo, los factores de confusión se equilibraron con respecto a las condiciones geográficas y sociodemográficas. Además, cada síntoma se ajustó según los posibles factores de confusión (edad, ocupación de la mujer, ocupación de la pareja y condiciones de la vivienda) mediante análisis de regresión logística. Sin embargo, tampoco puede descartarse la posibilidad de que existiera algún factor de confusión que no se haya tenido en cuenta por desconoci- 
miento o por error al clasificar algunos de los factores de análisis.

En el estudio se evaluaron numerosas variables, por lo que es posible que algunas de ellas dieran un resultado significativo al azar debido a las múltiples comparaciones. En casos así, podrían utilizarse procedimientos de inferencia múltiple. Sin embargo, la decisión de aplicar esos procedimientos es objeto de debate entre los estadísticos (21).

Para resumir, este estudio aporta pruebas de las adversas condiciones de salud que experimentaban las mujeres que vivían en la proximidad de pozos y estaciones de petróleo en la Amazonía ecuatoriana en el período de noviembre de 1998 a abril de 1999 . Las fuentes de agua de uso diario se encontraban intensamente contaminadas con hidrocarburos totales de petróleo. Después de ajustar por los factores de confusión, los síntomas que consideramos asociados con la exposición eran de esperar, según la literatura revisada. Sin embargo, se necesitan más estudios para confirmar estos resultados a la luz de otras comunidades expuestas a riesgos similares.

Es urgente formular y poner en práctica políticas y estrategias para controlar y remediar las condiciones ambientales y de salud relacionadas con la explotación petrolera. Esperamos que el gobierno del Ecuador, así como otros gobiernos, y las compañías petroleras sean capaces de elaborar conjuntamente planes para eliminar toda fuente de contaminación en la Amazonía ecuatoriana y otros lugares afectados, y que eviten así los innecesarios e inaceptables riesgos de salud a que están expuestas muchas poblaciones.

Agradecimientos. Agradecemos la colaboración de todas las participantes del estudio y el apoyo logístico de la Fundación Salud Amazónica y Sandi Yura. Reconocemos también el aporte de Paul Wilkinson en la revisión de este trabajo.

\section{REFERENCIAS}

1. Kimerling J. Amazon crude. New York: Brickfront Graphics Inc.; 1991.

2. Jochnick C, Normand R, Zaidi S. Rights violations in the Ecuadorian Amazon: the human consequences of oil development. Health Hum Rights 1994;1:82-100.

3. Kimerling J. Rights, responsibilities, and realities: environmental protection law in Ecuador's Amazon oil fields. Southwestern J Law Trade Americas 1995;2:293-384.

4. Unión de Promotores Populares de Salud de la Amazonía Ecuatoriana. Culturas bañadas en petróleo: diagnóstico de salud realizado por promotores. Quito: Abya-Yala; 1993.

5. Centro de Derechos Económicos y Sociales. Violaciones de derechos en la Amazonía Ecuatoriana. Quito: Abya-Yala; 1994.

6. Fundación Natura. La actividad petrolera en el Ecuador: aspectos ambientales y sociales. Quito: Fundación Natura; 1996.

7. Palinkas LA, Petterson JS, Russell J, Downs MA. Community patterns of psychiatric disorders after the Exxon Valdez oil spill. Am J Psychiatry 1993;150:1517-1523.

8. Campbell D, Cox D, Crum J, Foster K, Christie P. Initial effects of the grounding of the tanker Braer on health in Shetland. BMJ 1993;307: 1251-1255.

9. Campbell D, Cox D, Crum J, Foster K, Riley A. Later effects of grounding of tanker Braer on health in Shetland. BMJ 1994;309:773-774.

10. Lyons RA, Temple MF, Evans D, Fone DL, Palmer SR. Acute health effects of the Sea Empress oil spill. J Epidemiol Community Health 1999;53:306-310.

11. Morita A, Kusaka Y, Deguchi Y, Moriuchi A, Nakanaga Y, Masayuki I, et al. Acute health problems among the people engaged in the cleanup of the Nakhodka oil spill. Environ Res 1999;81:185-194.
12. Olin RG, Ahlbom A, Lindberg-Navier I, Norell SE, Spannare B. Occupational factors associated with astrocytomas: a case-control study. Am J Ind Med 1987;11:615-625.

13. Pan BJ, Hong YJ, Chang GC, Wang MT, Cinkotai FF, Ko YC. Excess cancer mortality among children and adolescents in residential districts polluted by petrochemical manufacturing plants in Taiwan. J Toxicol Environ Health 1994;43:117-129.

14. Yang CY, Chiu HF, Chiu JF, Kao WY, Tsai SS, Lan SJ. Cancer mortality and residence near petrochemical industries in Taiwan. J Toxicol Environ Health 1997;50:265-273.

15. San Sebastián M. Informe Yana Curi: impacto de la actividad petrolera en la salud de poblaciones rurales de la Amazonía ecuatoriana. Quito: Abya-Yala; 2000.

16. Zehner R, Villacreces LA. Estudio de la calidad de aguas de río en la zona de amortiguamiento del Parque Nacional Yasuní. primera fase: monitoreo de aguas-screening octubre de 1997. Coca, Ecuador: Laboratorio de Aguas y Suelos P. Miguel Gamboa-Fepp; 1998.

17. Cabodevilla MA. Coca: la región y sus historias. Pompeya, Ecuador: Cicame; 1997.

18. Cox DR, Snell EJ. Applied statistics: principles and examples. London: Chapman and Hall; 1981.

19. Ozonoff D, Boden LI. Truth and consequences: health agency responses to environmental health problems. Science Technol Hum Values 12(3-4):70-77.

20. Pershagen G. Interpretation of epidemiological studies with modestly elevated relative risks. En: Bate R, ed. What risk? Oxford: Butterworth-Heinemann. Pp. 191-200.

21. Rothman KJ, Greenland S. Modern epidemiology. Philadelphia: Lippincott-Raven; 1998.
22. Stata Corporation. Stata Reference Manual. Texas: Stata Press; 1999.

23. Garzón P. Impacto socioambiental de la actividad petrolera: estudio de caso de las comunidades San Carlos y La Primavera. En: Varea A, Ortiz P, eds. Marea negra en la Amazonía: conflictos socioambientales vinculados a la actividad petrolera en el Ecuador. Quito: Abya-Yala; 1995. Pp. 265-294.

24. Fabra A. Indigenous peoples, environmental degradation and human rights: a case study. En: Boyle A, Anderson M, eds. Human rights approaches to environmental protection. Oxford: Clarendon Press; 1998. Pp. 245-264.

25. Ministerio de Medio Ambiente. Informe de inspección ambiental al área de las comunidades Flor de Manduro y Centro Manduro ubicadas en el bloque siete operado por la compañía Oryx. Quito: Ministerio de Medio Ambiente; 1999.

26. Goldstein DH, Bendit JN. An epidemiological study of an oil mist exposure. Arch Environ Health 1970;21:600-603.

27. Kaplan MB, Brandt-Rauf P, Axley JW, Shen TT, Sewell GH. Residential release of number 2 fuel oil: a contributor to indoor air pollution. Am J Public Health 1993;83(1): $84-88$.

28. Kroeger A. South American Indians between traditional and modern health services in rural Ecuador. Bull Pan Am Health Organ 1982;16(3):242-254.

29. Kunst A. Cross-national comparisons of socio-economic differences in mortality [tesis de doctorado]. Rotterdam: Department of $\mathrm{Pu}-$ blic Health. Erasmus University; 1997.

30. Department of Health. Our healthier nation. London: Stationery Office; 1998.

31. Berkman L, Kawachi I. Social epidemiology. Oxford: Oxford University Press; 2000. 
32. Frerichs RR, Becht JN, Foxman B. A household survey of health and illness in rural Bolivia. Bull Pan Am Health Organ 1980;14(4): 343-355.

33. Ross DA, Vaugham JP. Health interview surveys in developing countries. Evaluation and Planning Centre for Health Care. London:
London School of Hygiene and Tropical Medicine; 1984.

34. Ozonoff D, Colten ME, Cupples A, Heeren T, Schatzkin A, Mangione T, et al. Health problems reported by residents of a neighbourhood contaminated by a hazardous waste facility. Am J Ind Med 1987;11(5):581-597.
Manuscrito recibido el 13 de julio de 2000. Aceptado para publicación, tras revisión, el 12 de abril de 2001.

Objective. Investigate the environmental conditions in and the state of health of women who live in rural communities surrounded by oil wells and oil production stations in the Amazon region of Ecuador.

The health of women who live near oil wells and oil production stations in the Amazon region of Ecuador
Method. We used a comparative cross-sectional design, classifying exposure according to the location of the communities with respect to the oil wells and production stations. Water samples from the local rivers were analyzed to determine total petroleum hydrocarbons, and a structured questionnaire was used with the head of each family in the study. The study was performed in rural communities in northeastern Ecuador from November 1998 through April 1999. The study included 9 communities in the exposed area (368 participants) and 14 communities in the unexposed area (291 participants).

Results. The rivers of the exposed communities showed contamination levels much above the limits acceptable for human use. Statistically significant differences between the exposed communities and the unexposed communities were found for the prevalence of skin fungi for the two weeks prior to the study (odds ratio $(\mathrm{OR})=1.37$; $95 \%$ confidence interval $(\mathrm{CI})=1.01-1.86)$ as well as for nasal irritation $(\mathrm{OR}=2.18,95 \%$ $\mathrm{CI}=1.64-2.91)$ and for throat irritation $(\mathrm{OR}=1.68,95 \% \mathrm{CI}=1.02-2.75)$ in the preceding 12 months. Also associated with exposure in the two preceding weeks were fatigue and the category of "other symptoms"; similarly associated with exposure in the preceding 12 months were headaches, eye irritation, earaches, diarrhea, and gastritis. Conclusions. The symptoms found among the participants in the exposed communities match the toxicity symptoms caused by oil. There is an urgent need to establish an adequate environmental control and remediation program in order to prevent unnecessary and unacceptable health hazards for these populations.

\section{Erratum \\ Instantánea "Una dieta sana y el ejercicio pueden reducir el riesgo de diabetes tipo 2", Vol. 9, No. 5, pp. 323-324}

La redacción de la Revista/Journal advierte a los lectores que se ha cometido un error en el número de mayo (Vol. 9, No. 5) de 2001, p. 324, línea 10, columna izquierda. Las páginas indicadas (1343-1352) no corresponden al editorial "Changing habits to delay diabetes" de Tataranni PA y Bogardus C, sino a un artículo titulado "Prevention of type 2 diabetes mellitus by changes in lifestyle among subjects with impaired glucose tolerance", de Tuomilehto $\mathrm{J}$, Lindstrom J, Eriksson JG, Valle TT, Hamalainen H, Ilanne-Parikka P, et al., que fue publicado en el mismo número de $N$ Engl J Med (2001;344:18). El editorial, que es la fuente de la instantánea, se ubica en las páginas 1390-1392.

En el presente número de la Revista/Journal los lectores podrán leer una instantánea sobre el artículo de Tuomilheto et al. 